\title{
Analysis of a Survey Database of Pet Food-Induced Poisoning in North America
}

\author{
Wilson K. Rumbeiha • Dalen Agnew • Grant Maxie • \\ Brent Hoff • Connie Page • Paul Curran • \\ Barbara Powers
}

Published online: 15 April 2010

(C) American College of Medical Toxicology 2010

\begin{abstract}
Following the outbreak of pet food-induced nephrotoxicity in March 2007, a voluntary online survey of all AAVLD-accredited laboratories, commercial laboratories, and veterinary clinics across North America was conducted. There was no information on toxicity of melamine or factors affecting the disease outcome following exposure to melamine in pets. Data were collected from affected pets to learn about the disease outcome and the affected pet population. The web-based electronic survey used the online tool, SurveyMonkey ${ }^{\mathrm{TM}}$. Data were collected between April 5 and October 31, 2007. Four hundred fifty-one cases of 586 reported cases met the criteria for inclusion in the study. Most reported cases were from California, Texas, Michigan, Florida,
\end{abstract}

W. K. Rumbeiha $(\bowtie) \cdot$ D. Agnew

Diagnostic Center for Population and Animal Health, College of Veterinary Medicine, Michigan State University,

4125 Beaumont Road,

Lansing, MI 48910-8104, USA

e-mail: rumbeiha@dcpah.msu.edu

G. Maxie $\cdot$ B. Hoff

Animal Health Laboratory, Laboratory Services Division,

University of Guelph,

P. O. Box 3612, Guelph, ON, Canada, N1H 6R8

C. Page $\cdot$ P. Curran

Department of Statistics and Probability,

Michigan State University,

East Lansing, MI 48823, USA

B. Powers

Veterinary Diagnostic Laboratory, Colorado State University, Fort Collins, CO 80523, USA and Ontario. Of the 451 cases, 424 were reported as affected. Of these, 278 cases $(65.6 \%)$ were cats and 146 (34.4\%) were dogs. A total of 278 pets (171 cats and $107 \mathrm{dogs}$ ) were reported to have died (a ratio of 1.6:1). However, within species, there was a higher percentage of deceased dogs $(73.3 \%)$ than cats $(61.5 \%)$. Of the affected pet population, older male cats with preexisting disease conditions were more likely to be deceased. Analysis of the pets in this large database of naturally affected pets yielded interesting findings. It showed that more cats than dogs were affected and also that preexisting renal diseases and old age predicted the most severe outcome (death or euthanasia) than any other factors.

Keywords Melamine $\cdot$ Pet food-induced toxicity Melamine-induced nephrotoxicity

\section{Introduction}

In March and April of 2007, a massive recall of melamine-contaminated pet food products occurred across North America, prompted by reports of acute renal failure among pets that had consumed tainted pet food containing ingredients imported from China. Illnesses and deaths among pets were first noticed in early March 2007 and were initially associated with feeding pet foods manufactured by Menu Foods, Inc. The first nationwide recall of pet foods was issued on March 16, 2007 [1]. Investigations to detect possible food contaminants focusing on common nephrotoxicants (ethylene 
glycol, soluble oxalates, mycotoxins such as ochratoxins, and heavy metals such as arsenic and mercury) were fruitless. Despite intensive efforts, it was not until March 30, 2007 that the first contaminant, melamine, was officially identified in pet foods sickening and killing pets. Reports surfaced that two Chinese companies had sold wheat gluten and rice gluten purposefully contaminated with melamine, an industrial chemical, in order to artificially inflate their apparent protein concentration. Melamine, which has no nutritional value but a high nitrogen content, is used for the manufacture of plastic resins used in kitchenware, for manufacture of glues and adhesives, and in Asia, as a fertilizer [2]. Wheat and rice gluten are used in the manufacture of wet foods as thickening agents. In 2008, thousands if infants in China were poisoned by melamine-contaminated milk food products. As such, the toxicity of melamine and other related triazine compounds is of interest to both medical and veterinary professionals [3].

Melamine is known to be of low toxicity in mammals $[2,4]$. The oral $\mathrm{LD}_{50}$ in the rat is $3,161 \mathrm{mg} / \mathrm{kg}$ body weight [2]. In dogs and rats, melamine given in high acute oral doses causes diuresis [5]. Chronic exposure of rats to melamine is associated with tumors of the urinary bladder and not the acute renal failure that was observed in pets [6-8]. The concentration of melamine found in pet food was relatively low (50-400 ppm). Based on all this, it became clear that other contaminants could be present in the pet food.

Further analysis of contaminated food in April 2007 revealed the presence cyanuric acid, ammeline, and ammelide; still later ureidomelamine and $N$-methylmelamine were also found [9]. Of these contaminants, melamine was the most abundant followed by cyanuric acid [9]. The wheat gluten was found to contain melamine at $8.4 \%$, cyanuric acid at $5.3 \%$, ammelide at $2.3 \%$, ammeline at $1.7 \%$, and ureidomelamine and methyl melamine at $<1 \%$ each [9]. All these contaminants are triazine compounds that have wide industrial applications. Cyanuric acid, ammelide, and ammeline appear to be by-products generated in the process of manufacturing melamine.

Cyanuric acid by itself, like melamine, has very low toxicity [10]. However, when melamine and cyanuric acid are ingested together, they interact in the renal tubules to form stable melamine-cyanurate crystals [11]. These crystals have very low water solubility, and renal failure may result from physical blockage of the uriniferous tubules and tubular epithelial cell necrosis [11].

Affected pets suffered mainly from acute renal failure characterized by polyuria, isosthenuria, polydipsia, oliguria, hyperphosphatemia, and azotemia [8]. The melaminecyanurate crystals found in the urine of these animals have been characterized recently [12].
At the time of the disease outbreak, very little had been published regarding the toxicity of triazine compounds in pets, and there was no information on the toxicity of a mixture of these compounds. The volume of unsubstantiated reports of pets having been affected and/ or having died as result of consuming melaminecontaminated food was exacerbated by confusion surrounding the case definition of the pet food-induced illness or death [13]. Through its national organizations, the veterinary professional community worked to develop information about this new disease in order to assist affected pet owners and veterinarians in its proper identification and appropriate treatment.

This survey was posted online on April 5, 2007, 1 week after melamine was identified in the pet foods. The major objectives of the survey were to collect data from veterinary professionals across North America to determine (a) the minimum number of confirmed cases of pets affected by melamine-contaminated food, (b) the geographic distribution of cases across North America, (c) the common signalment among affected animals, and (d) to determine the disease outcome among confirmed cases in this outbreak.

\section{Materials and Methods}

\section{Survey}

The web-based electronic survey used the online tool, SurveyMonkey ${ }^{\mathrm{TM}}$. The initial target audience was veterinarians in AAVLD-accredited laboratories, but the scope rapidly expanded to include those in commercial laboratories and veterinary clinics across North America. The online survey was widely publicized through the American Veterinary Medical Association (AVMA), the American College of Veterinary Pathologists (ACVP), and the American College of Veterinary Internal Medicine (ACVIM), and links were created on the AAVLD, AVMA, and ACVP websites. More publicity was generated by direct e-mails through the veterinary toxicology list serve, the news media, and conference presentations at the annual AAVLD meeting in Reno, Nevada. The survey period was April 5 through October 31,2007 . The 17-item questionnaire is reproduced in Appendix.

\section{Data}

Responses were received from 474 veterinarians reporting 586 individual pets. In all, 135 cases $(23 \%)$ were excluded for the following reasons: 13 responses failed to report species, five did not report health status of the 
animals, and 117 included no contact information or did not want to be contacted. Because this study targeted affected animals, another 27 cases were removed since they report the animal as "healthy, never ill." The remaining 424 reported cases were evaluated.

\section{Measurements}

The measurements discussed here are used in the "Results" section. The question number on the survey is also referenced when applicable. The following animal characteristics were asked directly on the survey (see Appendix): Q1, species (canine, feline, or other); Q2, breed (enter name); Q3, age (reported in years/months); and Q4, sex (male or female).

The survey asked (Q5), "What is the current health status of the affected animal?" The possible responses were healthy never ill, "currently ill," "previously ill, now recovered," and "deceased." For this study, we defined an animal as "affected" if the response to the current health status was currently ill, previously ill now recovered, or deceased. Only the 424 affected animals are used in any analysis, which compares recovered cases to deceased cases, as recovery requires onset of an illness from which recovery was made. Furthermore, a dichotomous variable, deceased, is defined as either not deceased if currently ill, previously ill now recovered, or otherwise deceased. This variable is used in one of the prediction models.

The survey asked (Q7), "Did this animal have any conditions that would predispose it to renal disease/failure?" The response was yes or no. This is a veterinarian's professional assessment of whether or not the pet had preexisting diseases that might render it more vulnerable to pet food-induced renal failure. For those who responded yes, selections from a list of predisposing conditions could be made (Q8). Multiple selections were allowed and respondents also given a choice of "other" where they freely listed those predisposing conditions, which were not provided as a choice.

The survey asked (Q9) "Please indicate diagnostic criteria used to arrive at your diagnosis (select all that apply)." The respondents could select from a list of eight criteria plus other. Respondents who checked other were asked to specify the other diagnostic criteria they used (see Q9, Appendix). Veterinarians were asked (Q10) to specify other chemical markers present in their cases, which was another way of asking about other diagnostic criteria they used to determine that the case they were entering was indeed pet food related.

The survey asked (Q11) "How soon after ingesting recalled pet food did the animals become ill?" Respondents chose from a list of five choices plus other. Those who chose other were asked to specify their answers.
The survey asked (Q12), "Which dog/cat food(s) was the affected animal eating at the time of (or immediately preceding) the diagnosis? (Select all that apply)." The respondent could select from a list of food brands or specify particular brands not on the selection list under other. When other was entered, the brand was added to the final list of reported food brands. See Q12, Appendix, for the final list. Participants were able to check multiple food brands that were fed to the affected pet, if this were the case, so each case may have values in more than one category. Each category is treated as yes/ no response. The rest of the questions (Q13-17) were designed to collect pet identification information, specific food lot numbers, and for identification of veterinarians completing the survey, their practice addresses, and contact information in case a follow-up was necessary.

\section{Statistical Analysis}

Diagnostic criteria (Q9) were analyzed for all pets. Statistical analyses focused on affected pets (defined by responses currently ill, previously ill now recovered, or deceased on the health status question, Q5). Univariate descriptive statistics for each species are presented for the animal characteristics, health status, and preexisting conditions likely predisposing to renal failure. Then, also for affected pets, bivariate analyses using cross-tabulation or comparison of means are performed to relate the animal characteristics, possible predisposition to renal disease, and health status.

Two predictive models are considered. A multinomial logistic model that considers the currently ill and previously ill now recovered categories separately and compares each category with deceased. A logistic model assesses risk factors related to renal failure for affected animals. Both model analyses are performed within species.

\section{Results}

Univariate Analysis for Affected Pets

The regional distribution of all reported, affected pets is summarized in Fig. 1. In North America, reports were submitted from four provinces, 35 states, and Puerto Rico, with the majority coming from California, Texas, Michigan, Florida, and Ontario. The USA reported $94.5 \%$ of the cases submitted, and the remaining $5.5 \%$ came from Canada.

Of the 424 affected pets, $278(65.6 \%)$ cases were cats and $146(34.4 \%)$ cases were dogs, with a cat/dog ratio of 1.9:1. 


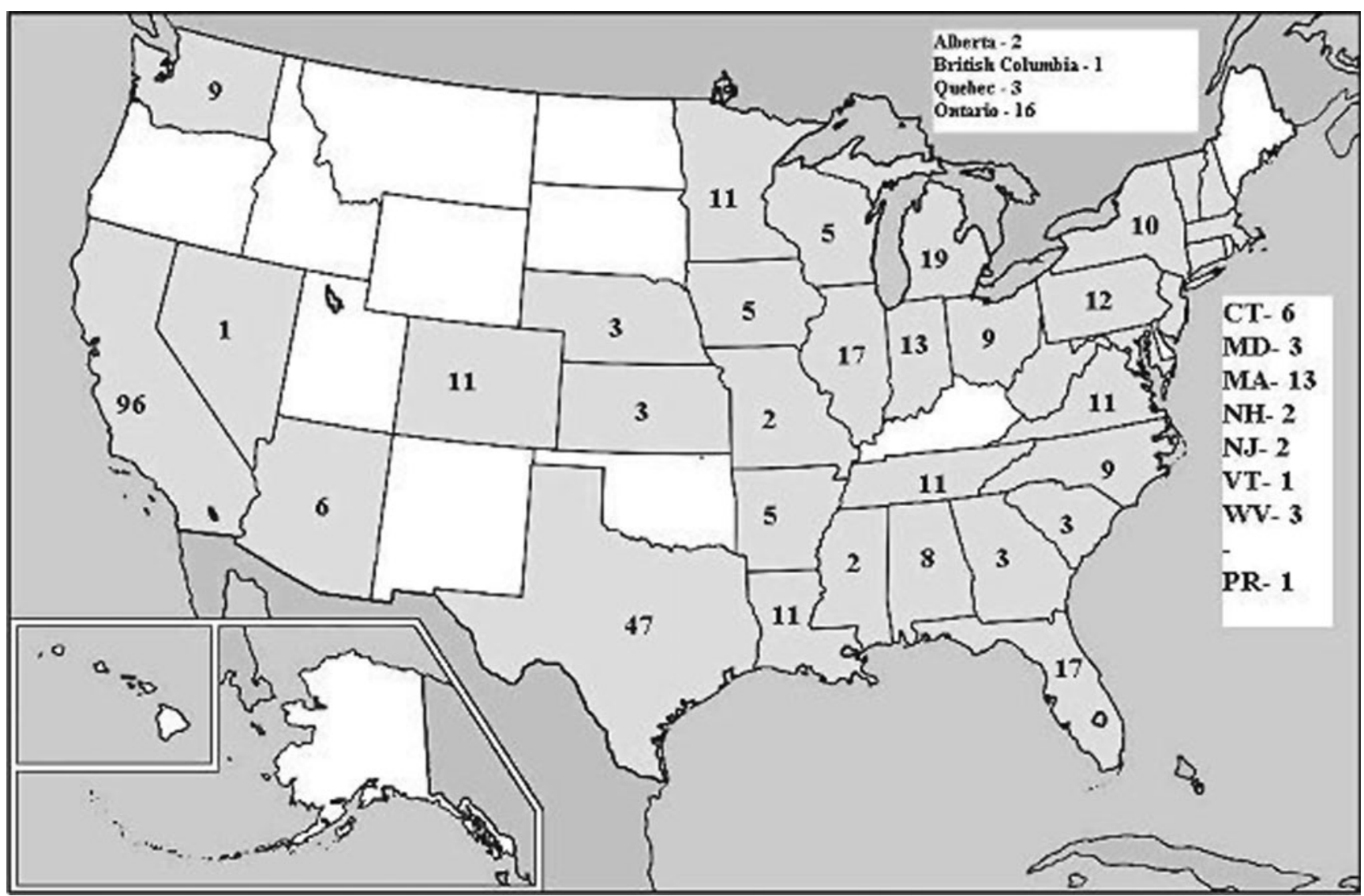

Fig. 1 Regional distribution of cases reported as affected in North America. Note that the states/provinces with most affected pets were California $>$ Texas $>$ Michigan $>$ Florida $>$ Ontario. Blank state $=$ no report

\section{Characteristics of Affected Pets}

Among the affected pets, 44 dog breeds and 14 cat breeds were represented. Dog breeds most frequently reported were crossbreeds > Labrador retrievers > Dachshund $>$ Pomeranian. Sixty-six percent of all cat cases were domestic short hair followed by the Siamese cat $(6.4 \%)$.

There was no significant difference for either dogs or cats in the number of cases reported for males and females $(p<0.35$; canine, $50.8 \%$ male and $49.2 \%$ female; feline, $47 \%$ male and $53 \%$ female).

The average age of affected cats was 92 months (7.6 years) with a standard deviation of 60 months (range, 2 to 252 months). The median age of affected cats was 84 months ( 7 years), and the median age was within 8 months of the average. The average age of affected dogs was 94 months (7.8 years) with a standard deviation of 54 (range, 4 to 228 months). The median age of affected dogs was 96 months ( 8 years), and the average and median ages were within 3 months of each other.
When sex is accounted for, the average age of affected female cats was 100 months $(n=135)$, while that of affected male cats was 82 months $(n=119)$. Using the two-sample $t$ test, the reported male cats were affected significantly younger than reported females $(p=0.015)$. For dogs, the average ages of affected females (93 months, $n=63$ ) and males ( 96 months, $n=64$ ) were not significantly different by $(p=0.695)$.

\section{Diagnostic Criteria}

A summary of diagnostic criteria (Q9) used for diagnosis of pet food-induced nephrotoxicity is given in Tables 1 (cats) and 2 (dogs). This question was asked to determine the criteria on which clinicians relied for diagnosis/ definition of those cases. The questions were not mutually exclusive, and respondents were told to mark all that applied and write in additional helpful parameters. Among deceased cats and dogs, the majority of veterinarians relied on a history of ingesting recalled pet food and on histopathology findings. In recovered or currently ill categories, the majority of respondents relied on the 
Table 1 Diagnostic criteria used for cats

\begin{tabular}{llll}
\hline Cats & Deceased (171) & Recovered (49) & Currently ill (58) \\
\hline Gross necropsy & $28(16.4 \%)$ & $3(6.1 \%)$ & $2(3.4 \%)$ \\
Histopathology & $121(70.8 \%)$ & 0 & $2(3.4 \%)$ \\
Urinalysis & $24(14 \%)$ & $13(26.5 \%)$ & $11(19 \%)$ \\
Melamine in food & $5(2.9 \%)$ & $1(2 \%)$ & 0 \\
Melamine in tissue & $4(2.3 \%)$ & 0 & 0 \\
Melamine in urine & $7(4.1 \%)$ & 0 & $1(1.7 \%)$ \\
Other marker in food, urine, or tissues & $11(6.4 \%)$ & $1(2 \%)$ & 0 \\
History of eating recalled pet food & $123(71.9 \%)$ & $45(91.8 \%)$ & $50(86.2 \%)$ \\
\hline
\end{tabular}

Note that multiple diagnostic criteria could be used. Note that for deceased cats, histopathology of the kidneys and a history of eating recalled food were the most commonly used criteria. For recovered and currently ill, urinalysis and history of ingesting recalled pet food were the most commonly used criteria. Not reflected in this table is "Serum chemistry profile" confirming azotemia, which was self-reported as widely used as one of the diagnostic criteria among recovered or currently ill

history of ingesting recalled pet food to make their diagnoses. Serum chemistry profiles indicative of azotemia were relied on by $41.7 \%$ of respondents. Additional diagnostic criteria listed included polydipsia, polyuria, low urinary specific gravity, renal biopsy, and ultrasound results consistent with hyperechoic corticomedullary junction. No response was received in response to Q10, "What other chemical marker was present in this case?"

\section{Predisposition to Renal Disease}

The majority of affected pets (330 of 419, 78.8\%) did not have any reported preexisting conditions that predisposed them to renal failure.

The conditions listed (gastrointestinal disease, renal disease, cardiovascular disease, neurological disease, cancer, and other) were not mutually exclusive, and $70 \%$ of those reporting preexisting conditions selected more than one condition in a given animal. By species, $22.5 \%$ of affected dogs $(n=142)$ and $20.6 \%$ of affected cats $(n=277)$ reported a predisposing conditions. Of the 19 positive canine responses, $15(78.9 \%)$ reported preexisting renal disease and four $(21.1 \%)$ reported cardiovascular disease. Of the 43 feline responses, 32 (74.4\%) reported preexisting renal disease, five $(11.6 \%)$ reported cardiovascular disease, and six (14.0\%) reported something outside of the list of categories. The most commonly reported preexisting renal diseases were diabetes, membranous glomerulonephritis, chronic interstitial nephritis and fibrosis, cancer (renal lymphoma and carcinoma), nephrosclerosis, unilateral kidney agenesis, and prior exposure to toxins such as Easter lilies (Q8).

Table 2 Reports of criteria for diagnosis (dog)

\begin{tabular}{llll}
\hline Dogs & Deceased (107) & Recovered (15) & Currently ill (24) \\
\hline Gross necropsy & $20(18.7 \%)$ & $1(6.7 \%)$ & $1(4.2 \%)$ \\
Histopathology & $69(64.5 \%)$ & 0 & $2(8.3 \%)$ \\
Urinalysis & $10(9.3 \%)$ & $5(33.3 \%)$ & $3(12.5 \%)$ \\
Melamine in food & $1(0.9 \%)$ & 0 & 0 \\
Melamine in tissue & $2(1.9 \%)$ & 0 & 0 \\
Melamine in urine & $2(1.9 \%)$ & 0 & $1(4.2 \%)$ \\
Other marker in food, urine, or tissues & $3(2.8 \%)$ & 0 & $1(4.2 \%)$ \\
History of eating recalled pet food & $78(72.9 \%)$ & $13(86.7 \%)$ & $17(70.8 \%)$
\end{tabular}

Note that multiple diagnostic criteria could be used. Note that for deceased dogs, histopathology of the kidneys and a history of eating recalled food were the most commonly used criteria. For recovered and currently ill, urinalysis and history of ingesting recalled pet food were the most commonly used criteria. Not reflected in this table is "Serum chemistry profile" confirming azotemia, which was self-reported as widely used as one of the diagnostic criteria among recovered or currently ill 


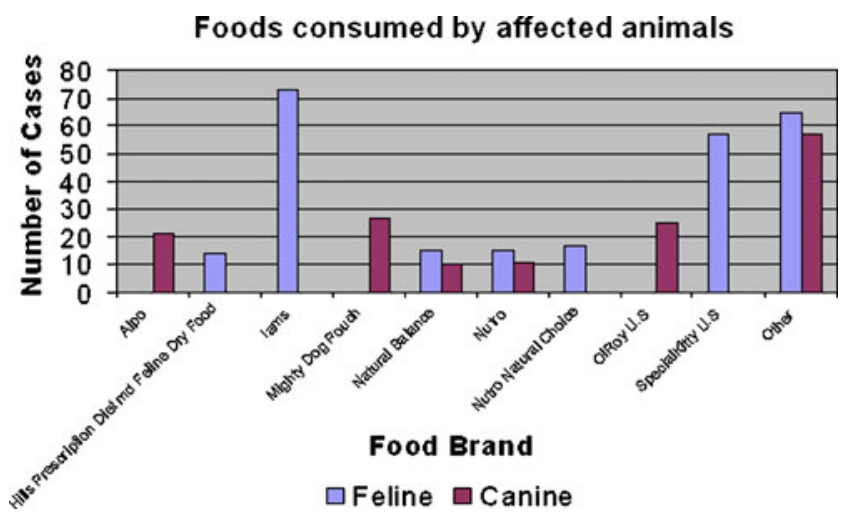

Fig. 2 Food brands consumed by affected pets. Note that for cats, Iams and Special Kitty were the two top brands that were consumed by affected cats. For dogs, the two top food brands were Ol' Roy US and Mighty Dog Pouch

\section{Foods Consumed Prior to Sickness}

In the USA, 20 food brands were reported to have poisoned dogs and 28 food brands for cats. In Canada, there were three food brands reported to have poisoned dogs and 10 food brands for cats. Figure 2 lists those food brands with a frequency of $>10$ cases reported, with the remainder included as other. Iams (Proctor \& Gamble) and Special Kitty (Menu Foods, Inc.) were the top brands most often consumed by affected cats, while Alpo (Nestle Purina), Mighty Dog Pouch (Nestle Purina), and Ol' Roy US (Menu Foods, Inc.) were those most often eaten by affected dogs.

Only 67\% of respondents addressed Q11, regarding the time interval between consumption and onset of illness. The majority of these (130 out of 394) indicated that the food brands were routinely fed, and it was unclear when a contaminated bag was opened. Of those who knew, four respondents indicated feeding less than 1 day, 69 respondents indicated 1 to 3 days, 74 indicated 3 to 7 days, 38 indicated 7 to 10 days, and 79 indicated more than 10 days. Overall, these results suggest that most of the cases occurred after multiple ingestions of the contaminated pet food.
Bivariate Analyses Relating Health Status and Predisposition to Other Variables

\section{Health Status Related to Other Variables}

The cross-tabulation of sex with health status showed no significant relationship for $\operatorname{dogs}(p=0.663)$ or for cats $(p=0.164)$. To compare the mean ages of affected pets in each health status category (currently ill, previously ill now recovered, and deceased), a one-way analysis of variance was run separately for cats and dogs (Table 3 ). For the affected animals, the mean age of the currently ill was higher for both dogs and cats, while the mean age for the recovered group is the lowest for both dogs and cats. However, the differences are not significant $(p=0.078)$.

The correlation of preexisting renal disease (Q7) with health status (Q5) is reported in Table 4. The association of preexisting renal disease with death is statistically significant in both dogs $(p=0.090)$ and cats $(p=0.023)$. For cats, $57.3 \%$ of those with no noted preexisting conditions died, compared to $77.2 \%$ of cats with known preexisting renal disease - renal impairment increased the incidence of death by $20 \%$. In dogs, $19.3 \%$ more animals died when preexisting renal conditions were reported $(87.5 \%$ vs $68.2 \%)$.

\section{The Relationship Between Preexisting Renal Disease (Q7) and Sex and Age}

The cross-tabulation of sex and whether the animal had any preexisting renal disease is reported in Table 5. The relationship is not significant for $\operatorname{dogs}(p=0.677)$, but is highly significant for cats $(p=0.007)$. These results imply that male cats were more likely than female cats to have preexisting renal diseases that rendered them more vulnerable to the melamine-cyanurate pet food-induced renal failure.

The two-sample $t$ test was used to analyze for differences in mean age between animals with any reported preexisting disease and those without preexisting conditions (Q7). For dogs, the mean age of the group with preexisting disease conditions was 106 months (8.9 years) with standard
Table 3 Mean age in months for each health status group

\begin{tabular}{llllll}
\hline Species & Health status & & & & \\
\cline { 2 - 3 } & Currently ill & Recovered & Deceased & & \\
\hline $\begin{array}{l}\text { Dogs }(N=133) \\
\text { Mean age (standard deviation) }\end{array}$ & $104.7(48.3)$ & $81.1(54.1)$ & $93.5(54.8)$ & 0.777 & 0.462 \\
$\begin{array}{l}\text { Cats }(N=268) \\
\text { Mean age (standard deviation) }\end{array}$ & $107.3(53.0)$ & $82.9(54.6)$ & $89.1(64.0)$ & 2.570 & 0.078 \\
\hline
\end{tabular}


Table 4 Health status by species and preexisting conditions

\begin{tabular}{lrlrl}
\hline & Currently ill & Previously ill, recovered & Deceased & $\chi^{2}, p$ value \\
\hline Cat & & & & \\
$\quad$ Preexisting conditions & $7(12.3 \%)$ & $6(10.5 \%)$ & $44(77.2 \%)$ & 0.023 \\
$\quad$ No preexisting conditions & $51(23.2 \%)$ & $43(19.5 \%)$ & $126(57.3 \%)$ & \\
Dog & & & & \\
$\quad$ Preexisting conditions & $3(9.4 \%)$ & $1(3.1 \%)$ & $28(87.5 \%)$ & 0.090 \\
$\quad$ No preexisting conditions & $21(19.1 \%)$ & $14(12.7 \%)$ & $75(68.2 \%)$ & \\
\hline
\end{tabular}

deviation of 59 months, and for the non-pre-existing disease group, the mean age was 89 months ( 7.4 years) with standard deviation of 52 months. The difference in mean age was not significant $(p=0.150)$. On the other hand, the mean age of the group of cats with preexisting disease conditions was 125 months (10.5 years) with standard deviation of 60 months, and for those without preexisting conditions, the mean age was 82 months (6.8 years) with standard deviation of 57 months. The difference in mean age is highly significant for cats, $p<0.001$. This means that older cats were more likely to have preexisting disease conditions predisposing them to melamine-contaminated pet food than younger cats or dogs. By far, the preexisting disease conditions were kidney-related (74.4\%).

Predictive Models

\section{Multinomial Logistic Model to Predict Death}

This model considers the currently ill and previously ill now recovered categories separately, comparing each category with deceased. The same predictor variables as in the logistic model are used. Results are reported in Table 6. For dogs, none of the variables are significant predictors of either currently ill vs deceased, or recovered vs deceased. However, for cats, age and predisposition to renal disease significantly distinguish currently ill from deceased, and predisposition is significant for recovered vs

Table 5 Percentage of each sex with preexisting conditions

\begin{tabular}{llrl}
\hline Species/sex & \multicolumn{2}{l}{ Preexisting conditions } & \multirow{2}{*}{$p$ value } \\
\cline { 2 - 3 } & Yes & No & \\
\hline Dog & & & \\
Male & $16(24.6 \%)$ & $49(75.4 \%)$ & 0.677 \\
Female & $14(21.5 \%)$ & $51(78.5 \%)$ & \\
Cat & & & \\
Male & $17(13.9 \%)$ & $105(86.1 \%)$ & 0.007 \\
Female & $38(27.7 \%)$ & $99(72.3 \%)$ & \\
\hline
\end{tabular}

deceased. A 1-year increase in age increases the odds of being currently ill rather than deceased by about $11 \%$. This is likely the result of the cross-sectional nature of the data since the final outcome for the ill animals is not known. However, the presence of preexisting predisposing diseases is a strong risk factor in death. Those cats with preexisting disease conditions have more than triple the odds of being deceased rather than currently ill, and more than 2.5 times the odds of being deceased rather than recovered. Although the average age of the recovered cats was slightly below the average age of deceased cats, the difference was not enough to conclude that age is a significant risk factor (comparing recovered and deceased) using this multinomial logistic model to predict death.

\section{Logistic Model to Predict Predisposition to Renal Disease}

Since preexisting disease plays an important role in survival, the question of whether it is a mediating factor is important. That is, can preexisting conditions predisposing to renal disease be predicted by sex and age? Results are reported in Table 7. For dogs, age and sex there are no significant findings. However, for cats, age and sex are significant risk factors in predisposition to renal disease. The odds ratio for sex is interpreted as the increase in odds of predisposition to renal disease when comparing males to females. Thus, for cats, the odds of predisposition to renal disease decrease by about $34 \%$ when the cat is female. In addition, for cats, the odds of predisposition to renal disease increase by about $14 \%$ for each year of age. This predisposition to renal disease, in turn, increases the chance of death or euthanasia. This suggests that following ingestion of melamine-cyanurate-contaminated pet food, older male cats were most at risk to die or be euthanized than any other segment of the pet population examined in this survey.

\section{Discussion}

This study was voluntary, and therefore, it was not possible to collect data on all pets that were affected by 
Table 6 Multinomial logistic regression with reference category "deceased"

\begin{tabular}{llll}
\hline Species & Variable & Odds ratio & $p$ value \\
\hline Dog & & & \\
Currently ill & Age in years & 1.10 & 0.143 \\
& Sex & 0.730 & 0.569 \\
& Preexisting predisposing diseases & 2.915 & 0.186 \\
Recovered & Age in years & 0.963 & 0.571 \\
& Sex & 0.617 & 0.400 \\
Cat & Preexisting predisposing diseases & 4.80 & 0.141 \\
Currently ill & & & $0.004^{*}$ \\
& Age in years & 1.11 & 0.074 \\
Recovered & Sex & 1.832 & $0.015^{*}$ \\
& Preexisting predisposing diseases & 3.21 & 0.806 \\
& Age in years & 1.01 & 0.481 \\
& Sex & 1.27 & $0.051^{*}$ \\
\hline
\end{tabular}

the melamine-cyanuric acid pet food contamination. The focus of the study was to determine the minimum number of confirmed cases of pet food-induced nephrotoxicity, to determine how geographically widespread the confirmed cases were, and, using this database of confirmed cases, to identify common factors, signalment, and disease outcome among clinically affected pets. In this web-based survey, 474 veterinarians responded reporting a total of 586 individual pets. As no control group was surveyed, a strong bias is undoubtedly present since only animals whose illness affected them enough to be noticed by the owners were likely presented to veterinarians. Many questions could only be answered descriptively in terms of the survey results. Inferential statistics were used where possible to extend predictions beyond the sample group.

Reported affected pets were fairly widely distributed throughout the USA, although no cases were reported in

Table 7 Logistic regression for predicting disposition to renal failure

\begin{tabular}{lcc}
\hline Species/variable & Odds ratio & $p$ value \\
\hline Dog & & \\
Age in years & 1.077 & 0.131 \\
Sex & 1.208 & 0.664 \\
Cat & & \\
Age in years & 1.142 & $<0.001^{* *}$ \\
Sex & 0.462 & $0.024^{*}$ \\
\hline
\end{tabular}

some states. This may be due to the uneven distribution of participating clinics or diagnostic laboratories. Respondents had an option to list species affected other than dogs and cats, but none were reported (Q1). Reports from experimental studies indicate that pigs and fish are also affected by melamine-cyanurate-contaminated food [14].

Results show that $65.5 \%$ of the affected pets were cats and $34.4 \%$ were dogs. According to the US Pet Ownership and Demographics Sourcebook information posted on the AVMA website, in 2007, there were 72.1 million dogs (in 43.0 million homes) and 81.7 million cats (in 37.5 million homes) in the USA [15]. Whereas there were proportionally more cats than dogs $(53.1 \%$ vs $46.9 \%)$, there were fewer homes with cats than with dogs $(46.5 \%$ vs $53.5 \%)$ in the USA in 2007, suggesting that more cats were kept per household. Comparing pet populations, more cats were affected than were represented in the general pet population ( $65.5 \%$ affected vs $53.5 \%$ in pet population) in 2007 . It is possible that more cats were affected than dogs because multiple cats in the same household may have been exposed to contaminated pet food. Alternatively, since published data show that daily dry matter intake in cats of $0.05-$ $0.8 \mathrm{~kg}$ animal $^{-1} \mathrm{day}^{-1}$ is similar to that of dogs $(0.07-$ $0.8 \mathrm{~kg}$ animal $^{-1}$ day $^{-1}$ ), it is possible that cats are more sensitive than dogs to melamine-cyanurate-contaminated pet food [16].

Among dogs, crossbreeds were the most frequently reported to have been affected, likely reflecting the overall popularity of mixed-breed dogs as pets. Unfortunately, animal weight was not included in the questionnaire; therefore, the size of these mixed dog breeds is 
unknown. Among the purebreds, the Labrador retriever, Dachshund, and Pomeranian were most represented. The Labrador retriever is a large breed, while the Pomeranian is a small dog. Dog size does not appear to be a factor as both large and small dogs were affected. However, the Labrador retriever is the most popular dog in the USA, and the results may simply reflect that fact. With respect to affected cat breeds, the domestic short hair cat was the most frequently affected. This is probably because it is the most common household breed of cat.

News reports indicated that "thousands" of pets died as a result of pet food-induced nephrotoxicity [13]. Results of this study show that the numbers of confirmed affected pets were at least 586 cases, but the total will probably never be known. Banfield, a large small animal practice in North America, indicated that only a small population of pets was actually affected by the contaminated pet food [17]. It is likely, however, that some pets had died before this outbreak was recognized and therefore were not reported. Furthermore, many affected pets may not have been presented for treatment, or for necropsy if deceased. It is also likely that despite the effort to publicize the survey as widely as was done, some veterinarians were not aware of it or chose not to participate.

Despite its shortcomings, this study yielded interesting data. Results show that overall within species, male and female pets were affected at about the same frequency. In addition, animals of all ages were affected, although the average and median age of affected pets was about 8 years old. A higher proportion of affected dogs died than affected cats, despite the observation that more cats than dogs were affected. The reasons for this are not clear. It is also noteworthy that the older male cat was most at risk to die or to be euthanized after ingesting contaminated pet food. It is not clear why older male cats experienced a more severe outcome than older female cats, this in spite of the finding that significantly more older female cats were affected than males.

Another interesting finding was that the majority of affected pets $(>78 \%)$ did not have preexisting diseases. This suggests that melamine cyanurate is a potent toxin that will affect even healthy pets. However, among pets reported to have preexisting conditions, $74.4 \%$ of cats and $78.9 \%$ of dogs had kidney-related diseases of which diabetes, glomerulonephritis, chronic interstitial nephritis, and kidney cancer were the most common. The results show that these preexisting diseases increased the incidence of death.

Many food brands were reported to have affected pets. However, some food brands were more frequently reported than others. For example, for cats, Iams and Special Kitty U.S. were two top foods eaten by affected pets. It is not clear if these food brands contained proportionally more contaminants than others, or if simply they were the most popular cat food brands. Also, the majority of veterinarians indicated that illness was observed in pets following several days of feeding contaminated pet food. This suggests cumulative effect(s) of the toxicants.

In conclusion, this study has shown that more cats than dogs were reported poisoned by the melaminecyanuric acid pet food contaminants. The proportion of affected cats to dogs was 1.9:1 compared to a ratio of 1.1:1 in the general population in 2007. Of those cases submitted in this survey, 278 pets were reported to have died. The cat to dog ratio of pets that died was 1.6:1. Within species however, a higher percentage of affected dogs $(73.3 \%)$ were reported to have died or euthanized compared to only $61.5 \%$ of cats. The vast majority of affected pets were healthy, without preexisting disease conditions. Of the pets reported to have preexisting conditions, the vast majority had preexisting renal diseases. Preexisting renal diseases and old age predicted the most severe outcome (death or euthanasia) than any other factors. Older male cats with preexisting diseases were the most vulnerable and more likely to die or be euthanized among pets that ate contaminated pet food.

Acknowledgments This study would not have been successful if there was no participation from veterinary clinicians and diagnosticians across North America. Special gratitude goes to diagnosticians who entered multiple cases including Drs. Nikos Gurfield (San Diego County Animal Disease Diagnostic Laboratory), Catherine Barr (TX), Jennifer Cobin (St Francis Veterinary Center), Tracy Darling (Cat Clinic Orange County), Pat Halbur (Iowa State University), Rebecca Harrod (Hazel Ridge Vet Clinic, CA), and Nikos Gurfield (San Diego County Diagnostic Lab). A special thank-you also goes to Liz Connelly who helped with the logistics of the survey instrument in April of 2007. Finally, we acknowledge all professional organizations such as the AAVLD, the AVMA, the ACVIM, and the ACVP for publicizing the survey.

Funding No funds were used on this project. The Diagnostic Center for Population and Animal Health, College of Veterinary Medicine, Michigan State University, allowed use of the software.

Previous presentation of data Preliminary results were presented at the annual AAVLD meeting in Reno Nevada in October 2008. These data have not been published or presented at another venue. 


\section{Appendix}

A Complete list of Questions on the Survey Questionnaire

1. What is the species of the affected animal?

Canine

Feline

Other (please specify)

2. What is the breed of the affected animal?

3. What is the age of the affected animal? Years Months

4. What is the sex of the affected animal? Male Female

5. What is the current health status of the affected animal?

Healthy never ill

Currently ill

Previously ill now recovered

Deceased

6. What geographic location was the affected animal living in at the time of illness onset?

Country

State or Province

7. In your opinion, did this animal have any conditions that would predispose it to renal disease/failure?

Yes

No

8. Define the predisposing condition:

Gastrointestinal disease

Renal disease

Cardiovascular disease

Neurologic disease

Cancer

Other (please specify)

9. Please indicate diagnostic criteria used to arrive at your diagnosis: (select all that apply)

Gross findings consistent with crystal-induced renal failure (with distinct ID of characteristic crystals) 
Histopathologic findings consistent with crystal-induced renal failure (with distinct ID of characteristic crystals)

Urinalysis consistent with characteristic crystals

Analytical confirmation of melamine marker in food

Analytical confirmation of melamine marker in animal tissues

Analytical confirmation of melamine marker in urine

Analytical confirmation of any chemical marker in tissue, urine, or food.

History of having eaten recalled pet food

Other (please specify)

10. What other chemical marker was present in this case?

11. How soon after ingesting the recalled pet food did the animal become ill?

Less than 1 day

1 to 3 days

3 to 7 days

7 to 10 days

More than 10 days

Other (please specify)

12. Which $\mathrm{dog} / \mathrm{cat}$ food(s) was the affected animal eating at the time of (or immediately preceding) the diagnosis? (Select all that apply)

Alpo

AmericasChoicePreferredPets

Authority

Award

BestChoice

BigBet

BigRed

Bloom

Cadillac

Companion

\section{Compliments}

DemoulasMarketBasket

Eukanuba

FineFelineCat

FoodLion

Foodtown

GiantCompanion

GreatChoice

Hannaford

HillCountryFare 
HillsPrescriptionDietmdFelineDryFood

HyVee

Iams

LauraLynn

LilReed

LovingMeals

MeijersMainChoice

MightyDogPouch

NaturalBalance

Mixables

Nutriplan

Nutro

NutroMax

NutroMaxGourmetClassics

NutroNaturalChoice

NutroUltra

OlRoyCanada

OlRoyU.S

Paws

PetEssentials

PetPride

PetPrideGoodnMeaty

PresidentsChoice

PriceChopper

PriorityCanada
PriorityU.S

Purina

Publix

RocheBrothers

SaveaLotChoiceMorsels

SavaLotSpecialBlend

Schnucks

ScienceDietFelineSavoryCutsCans

ShepDog

Sophistacat

SpecialKittyCanada

SpecialKittyU.S

SpringfieldPrize

Sprout

StaterBrothers

StopShopCompanion

TopsCompanion

Wegmans

WegmansBruiser

WeisTotalPet

WesternFamilyU.S

WhiteRose

WinnDixie

YourPet

Other_3

13. Dog/cat food lot number (if known)

14. Dog/cat food UPC (Universal product code) number (if known)

15. Please provide the unique animal-identification number for this case. 
16. Can we contact you regarding this case if further review is deemed beneficial?

Yes

No

17. Please provide contact information:

\section{Contact name}

Institution

\section{Address 1:}

Address 2:

Contact Phone number

Contact e-mail

\section{References}

1. Burns K, Nollen S, Kahler S, Rezendies A (2007) Recall of pet food leaves veterinarians seeking solutions. JAVMA 230 (12):1795, April 15, 2007 News

2. OECD (2002) SIDS analysis, melamine. UNEP, Nairobi, pp 111198

3. Hau KA, Kwan TH, Li PK (2009) Melamine toxicity and the kidney. J Am Soc Nephrol 20:245-250

4. Clark R (1966) Melamine crystalluria in sheep. J S Afr Vet Med Assoc 37:349-351

5. Lipschitz WI, Stokey E (1945) The mode of action of three new diuretics: melamine, adenine and formoguanamine. J Pharmacol Exp Ther 83:235-249

6. Melnick RL, Boorman GA, Haseman JK, Montali RJ, Huff J (1984) Urolithiasis and bladder carcinogenicity of melamine in rodents. Toxicol Appl Pharmacol 72:292-303

7. Ogasawara $\mathrm{H}$, Imaida $\mathrm{K}$, Ishiwata $\mathrm{H}$, Toyoda $\mathrm{K}$, Kawanishi $\mathrm{T}$, Uneyama C et al (1995) Urinary bladder carcinogenesis induced by melamine in F344 male rats: correlation between carcinogenicity and urolith formation. Carcinogenesis 16:2773-27777

8. Puschner B, Poppenga RH, Lowenstine LJ, Filigenzi MS, Pesavento PA (2007) Assessment of melamine and cyanuric acid toxicity in cats. J Vet Diagn Invest 19:616-624

9. Dobson RLM, Motlagh S, Quijano M, Cambron T, Baker RT, Pullen MA et al (2008) Identification and characterization of toxicity of contaminants in pet food leading to an outbreak of renal toxicity in cats and dogs. Tox Sci 106:251-262

10. Hodge HC, Panner BJ, Downs WL, Maynard EA (1965) Toxicity of cyanurate. Toxicol Appl Pharmacol 17:667-674

11. Brown CA, Jeong K, Poppenga RH, Puschner B, Miller DM, Ellis $\mathrm{AE}$ et al (2007) Outbreaks of renal failure associated with melamine and cyanuric acid in dogs and cats in 2004 and 2007. J Vet Diagn Invest 19:525-531

12. Thompson ME, Lewen-Smith MR, Kalasinsky VF, Pizzolato M, Fleetwood ML, McElhaney MR et al (2008) Characterization of melamine-containing and calcium oxalate crystals in three dogs with suspect pet food-induced nephrotoxicosis. Vet Pathol 45:417-426

13. New York Times (2007) Editorial. After the pet food contamination, May 7, 2007

14. Reimschuessel R, Gieseker CM, Miller RA, Ward J, Boehmer J, Rummel N et al (2008) Evaluation of the renal effects of experimental feeding of melamine and cyanuric acid to fish and pigs. Am J Vet Res 69:1217-1228

15. US pet ownership and demographics sourcebook 2007 edition. Cited 09/09/09. http://www.avma.org/reference/marketstats/ownership.asp on $09 / 09 / 09$

16. Puls R (1994) Water consumption and dry matter intake guidelines. In: Puls R (ed) Mineral levels in animal health. Sherpa International, Clearbrook, pp 307-307

17. Lundeen T (2007) Fewer pets ill from recall. Feedstuffs 79, April 16 , pp 1 and 5 